\title{
Zener tunneling in conductive graphite/epoxy composites: Dielectric breakdown aspects
}

\author{
L. X. He, S. C. Tjong* \\ Department of Physics and Materials Science, City University of Hong Kong, Hong Kong
}

Received 29 october 2012; accepted in revised form 3 January 2013

\begin{abstract}
The electrical responses of conductive graphite/epoxy composites subjected to an applied electric field were investigated. The results showed that reversible dielectric breakdown can easily occur inside the composites even under low macroscopic field strengths. This is attributed to the Zener effect induced by an intense internal electric field. The dielectric breakdown can yield new conducting paths in the graphite/epoxy composites, thereby contributing to overall electrical conduction process.
\end{abstract}

Keywords: polymer composites, electrical properties, physical methods of analysis, Zener effect

\section{Introduction}

Polymer composites with conducting particles such as metals and carbon blacks (CBs) find useful industrial applications in the fields of switching elements, sensors, actuators and electromagnetic shielding [1-4]. For these composites, the electrical conductivity $(\sigma)$ increases slowly with increasing filler concentration $(p)$, but then rises significantly when their filler content reaches a critical concentration, generally known as the percolation threshold $\left(p_{\mathrm{c}}\right)$. From the percolation theory, the relation between $\sigma$ and $p_{\mathrm{c}}$ is given by Equation (1) [5-7]:

$\sigma=\sigma_{0}\left(p-p_{\mathrm{c}}\right)^{\mathrm{t}}$

where $\sigma_{0}$ is the conductivity scale factor related to the intrinsic conductivity of the filler and $t$ the critical exponent depending on the dimensionality of the system, i.e. 1.6-2.0 for three dimensional, and 1.0-1.3 for two dimensional systems [7].

Percolative polymer composites usually display nonlinear electrical conduction behavior [8-12].
The application of electrical field in the composites can also lead to a nonlinear response. This is because the resistance of the composites changes from linear to nonlinear as the applied filed increases. When the field exceeds relatively large values, local joule heating of the elements occurs, causing irreversible damage in the structure of materials [13]. This is commonly referred to as the electrical failure or dielectric breakdown of the system. The irreversibility can be prevented if the current through the material is well-controlled. Alternatively, materials with nanoscale dimensions are reported to be effective for providing local heat sinks and preventing irreversible changes in the materials [14]. Therefore, dielectric breakdown of the polymer composites with conductive and nonconductive nanofillers has received considerable attention recently [14-17]. Very recently, Song et al. [16] reported factors influencing the breakdown strength of the ceramic oxide particle/polymer nanocomposites.

Nonlinear electrical transport can also occur in the composite materials by applying relatively small

\footnotetext{
${ }^{*}$ Corresponding author, e-mail: aptjong@cityu.edu.hk

(c) BME-PT
} 
electric field. The reversible nonlinear response is more complicated in the presence of tunneling conduction. The physical mechanisms responsible for such nonlinearity remain unclear. Sen et al. $[18,19]$ reviewed and analyzed nonlinear and dielectric breakdown of disordered composite materials systematically. Particular attention was paid to the reversible breakdown of the materials. In a previous study, we explored the effect of Zener tunneling in various carbon/polymer composites [20]. In this work, we attempt to establish a relationship for nonlinear electrical transport in the graphite/epoxy composites. We show that the reversible nonlinear behavior of the composite derives from local dielectric breakdown, or Zener tunneling.

\section{Experimental}

Graphite powder flakes of irregular shapes with an average size of $\sim 20 \mu \mathrm{m}$ (Product No. 332461, Sigma-Aldrich) and epoxy resin $(86.4 \%$ bisphenol and $13.6 \%$ N-butly glycidyl ether; Cat. No. Ultra3000R-128, Pace Technologies, Inc.) were used as conducting fillers and insulating matrix respectively. In a typical fabrication process, the epoxy was dissolved in acetone, followed by adding graphite powders. The suspension was sonicated for $2 \mathrm{~h}$ to ensure homogeneous dispersion of the graphite powders. Then the solution was kept at $50^{\circ} \mathrm{C}$ for $12 \mathrm{~h}$ for fully removal of acetone. This was followed by adding the hardener $(100 \%$ diethylenetriamine; Cat. No. Ultra-3000H-32, Pace Technologies, Inc.) to the graphite/epoxy mixture at a ratio of 1:10 by weight. The mixed liquid was stirred for $10 \mathrm{~min}$ in order to ensure homogeneous filler dispersion and to achieve good epoxy/hardener blending. Then the mixture was left in vacuum at room temperature for $24 \mathrm{~h}$. This led to full curing and crosslinking of the epoxy, yielding better dispersion of the fillers in the epoxy resin [21]. After curing, disk-like samples with a diameter of $10 \mathrm{~mm}$ and a thickness of about $1 \mathrm{~mm}$ were obtained. They were treated with silver paste to form the electrodes for the electrical measurements. A Hewlett Packard 4140B pA meter/DC voltage source with pulse testing voltage was used to measure the electrical responses. It required about 2-3 seconds for achieving the equilibrium DC conductivity. The voltage used to determine the DC conductivity was $500 \mathrm{mV}$. Ten samples were measured for each composite, and the obtained values were averaged. The scatter bars in the plot indi- cate the fluctuation of the conductivity. The dispersion of the graphite powders was examined using an optical microscope (OM, Olympus BH-2) in a transmission mode. The morphology of the composites was examined in a JSM 820 scanning electron microscope (SEM). The specimens for the microscopic observations were cut to about $20 \mu \mathrm{m}$ using a Reichert Ultra Cut S cutter.

\section{Results and discussion}

Figure 1 shows the optical micrographs of the fabricated graphite/epoxy composites. Figure 2 shows the SEM micrographs of several composite samples. The graphite powders are dispersed uniformly in the polymer matrix when the filler content $\leq 8 \mathrm{vol} \%$. At a higher filler loading (9.3 vol\%), the graphite powders tend to aggregate somewhat in the polymer matrix (see Figure 1f).

Figure 3 shows the plot of static conductivity $\sigma(p)$ against filler content for the samples studied. The conductivity follows the percolation theory as expected. By fitting the data to Equation (1), the percolation threshold $\mathrm{pc}$ and the critical exponent $\mathrm{t}$ are determined to be $4.8 \pm 0.6$ and $2.3 \pm 0.4$, respectively. When the graphite content reaches near $4.8 \mathrm{vol} \%$, an infinite conducting network spanning the whole system begins to form. And the static conductivity at this content is expected to show a sharp increase. As mentioned above, $t$ takes the value of 1.6-2.0 for three dimensional, and 1.0-1.3 for two dimensional system. However, it may become non-universal due to a large variation in the distribution of distances among conductive fillers within the polymer matrix [22]. Large $t$ values have also been reported in other polymer composite systems [23-25].

The current density $(J)$ as a function of electric field $(E)$ for these samples is shown in Figures $4 \mathrm{a}-4 \mathrm{f}$, respectively. These experimental data is highly reproducible and completely reversible, indicating no damage to the material by the electric field. However, the electrical conductivity of all samples rises with increasing field strength. This reversible conductivity is considered to be of particular interest. It may arise from two ways: in one case, the conducting elements are nonohmic while in another, the conducting elements are ohmic but their macroscopic conductivity becomes nonohmic due to the creation of additional channels for conduction [26]. Herein, we use a two dimensional random-bond model (Figure 5) to illustrate the conduction paths 


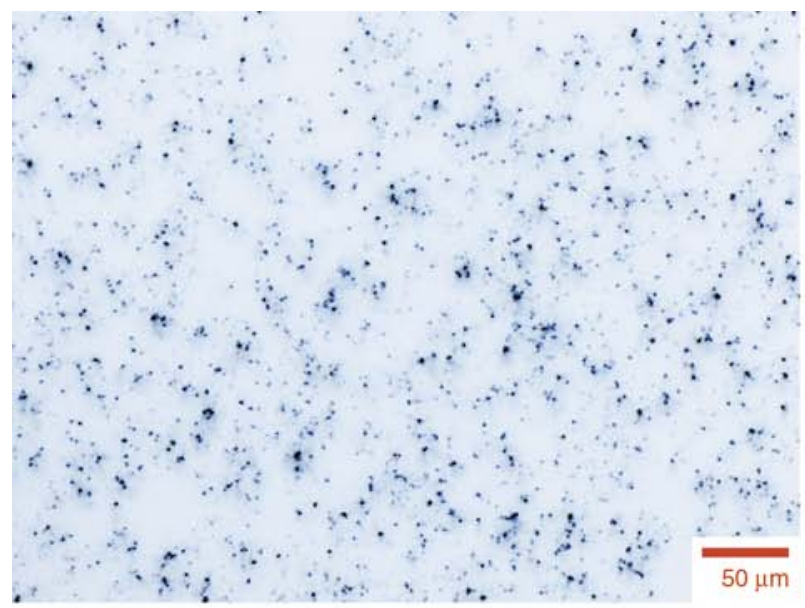

a)

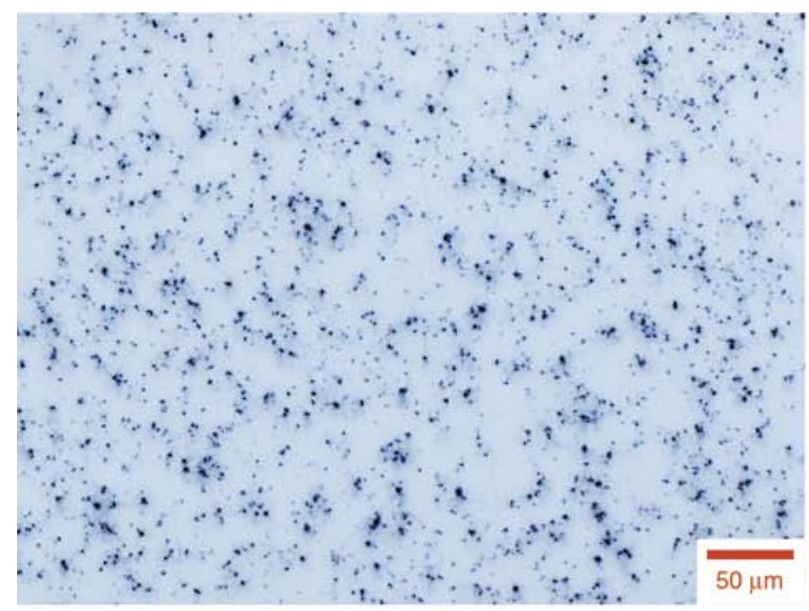

c)

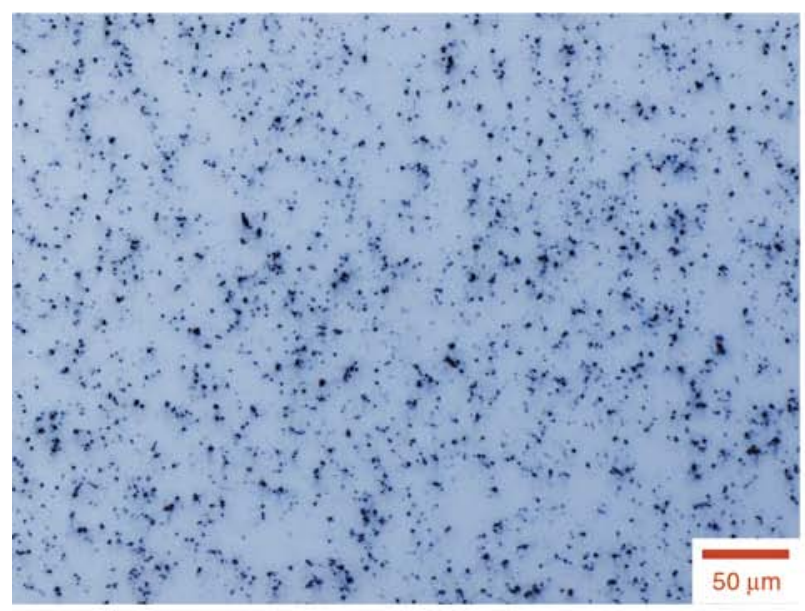

e)

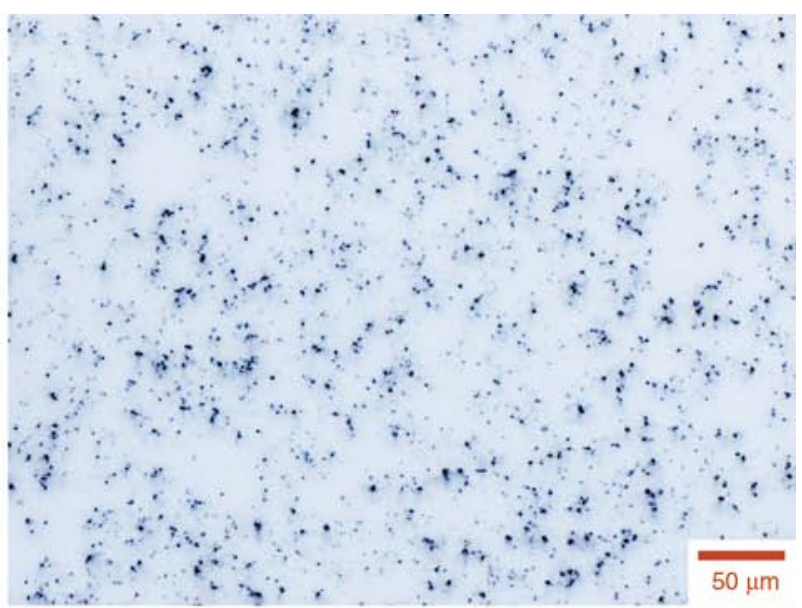

b)

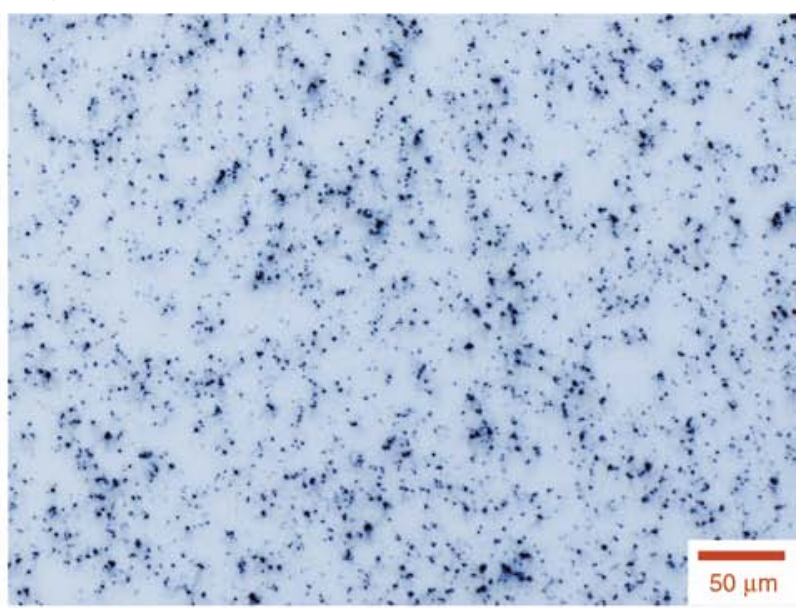

d)

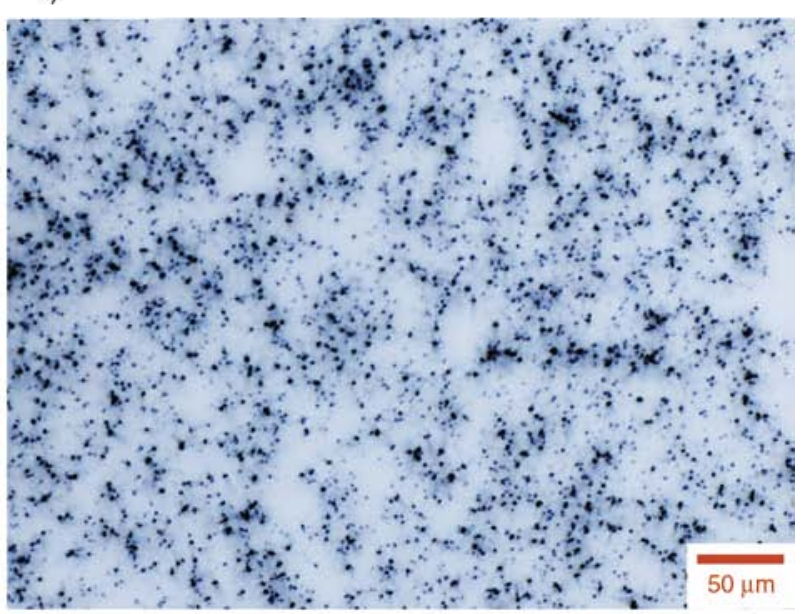

Figure 1. Transmission optical micrographs of graphite/epoxy composites with (a) 6 vol\%, (b) 6.4 vol $\%$, (c) 6.8 vol\%, (d) $7.4 \mathrm{vol} \%$, (e) $8 \mathrm{vol} \%$ and (f) $9.3 \mathrm{vol} \%$ filler content

involved in the graphite/epoxy composites. Because of the presence of the conducting clusters in the insulating matrix, local discontinuities in the field strength can be expected. For narrow insulating gaps between these clusters (1), the field strength is magnified by a factor $\gamma$ given by the ratio of the average size of the conducting clusters to the average gap width [27]. At the tips of these clusters, this magnified field concentrates locally to a large extent. Likewise, high electric field may also establish between different parts of the backbone (2), or between the backbone and the clusters (3). Therefore, it is proposed that micro dielectric breakdown would occur at these insulating layers. The conduction paths induced by dielectric breakdown would lead to an additional conducting network, i.e. a 


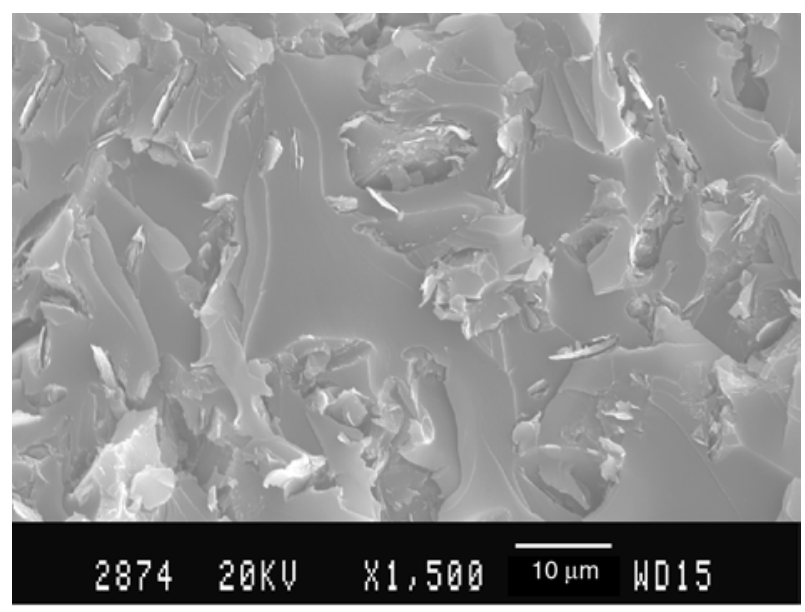

a)

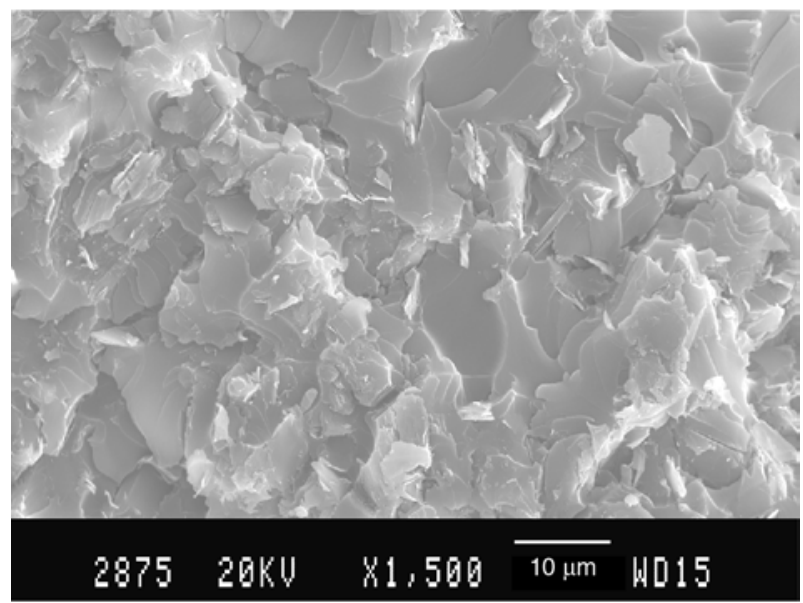

b)

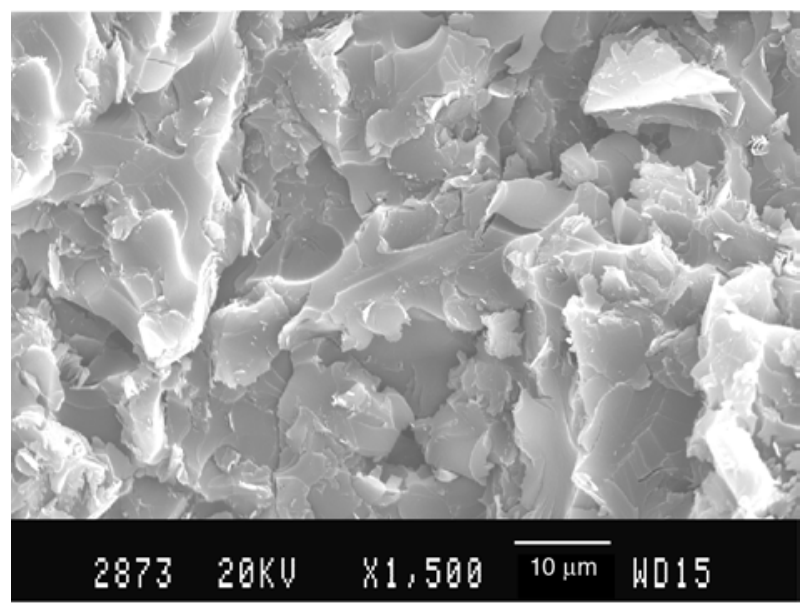

c)

Figure 2. SEM images of graphite/epoxy composites with (a) $6 \mathrm{vol} \%$, (b) $8 \mathrm{vol} \%$ and (c) $9.3 \mathrm{vol} \%$ filler content

breakdown network, which is responsible for the macroscopic dielectric breakdown. This network can lead to a nonlinear increase in the conductivity of the system.

The specific dielectric breakdown mechanism can be categorized into two types. The most common is avalanche breakdown, which takes place in extreme

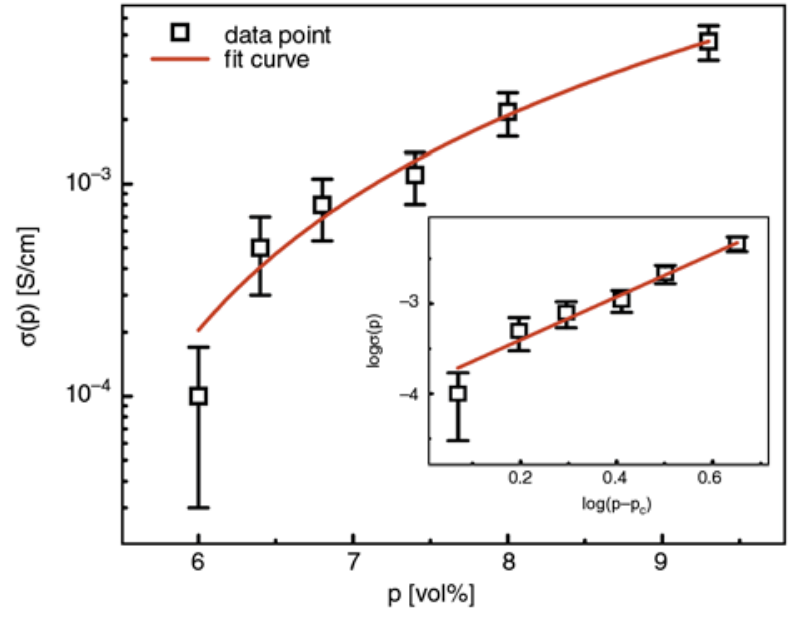

Figure 3. Static conductivity of the graphite/epoxy composites of various filler contents. The red solid lines are nonlinear fits to Equation (1). Ten samples were tested for each composite

conditions under the application of very large electric field. The avalanche breakdown is caused by an impact ionization that produces a large amount of charge carriers. This is often associated with a large current increment. To gain enough energy for ionizing the atoms, the electrons must move under a very strong electric field over a long distance. Avalanche breakdown usually refers to the permanent damage in insulators caused by a large electric field. Thus even the field strength decreases, the current still remains at a high level. In other words, the $J-E$ curve is irreversible. In the present study, it is observed that the current density rises moderately with increasing field strength, and all the $J-E$ curves are reversible (Figure 4). This excludes the possibility of avalanche breakdown. The second is the Zener breakdown, which involves the transitions of charge carriers between the valence and conduction band induced by appreciable electric fields [28]. It is also widely referred to as the interband tunneling, and commonly observed in semiconductor crystals [29], such as heavily doped $p-n$ junctions [30]. As recognized, conventional tunneling involves the transition of charge carriers over an energy barrier. The Fowler-Nordheim tunneling is associated with the pulling of electrons from a conductor to vacuum by an intense electric field. Zener tunneling involves the pulling of electrons from the valence band to the conduction band of an insulator, thus rendering it conductive by producing movable charge carriers. It can be regarded as a special form of Fowler-Nordheim tunneling. 


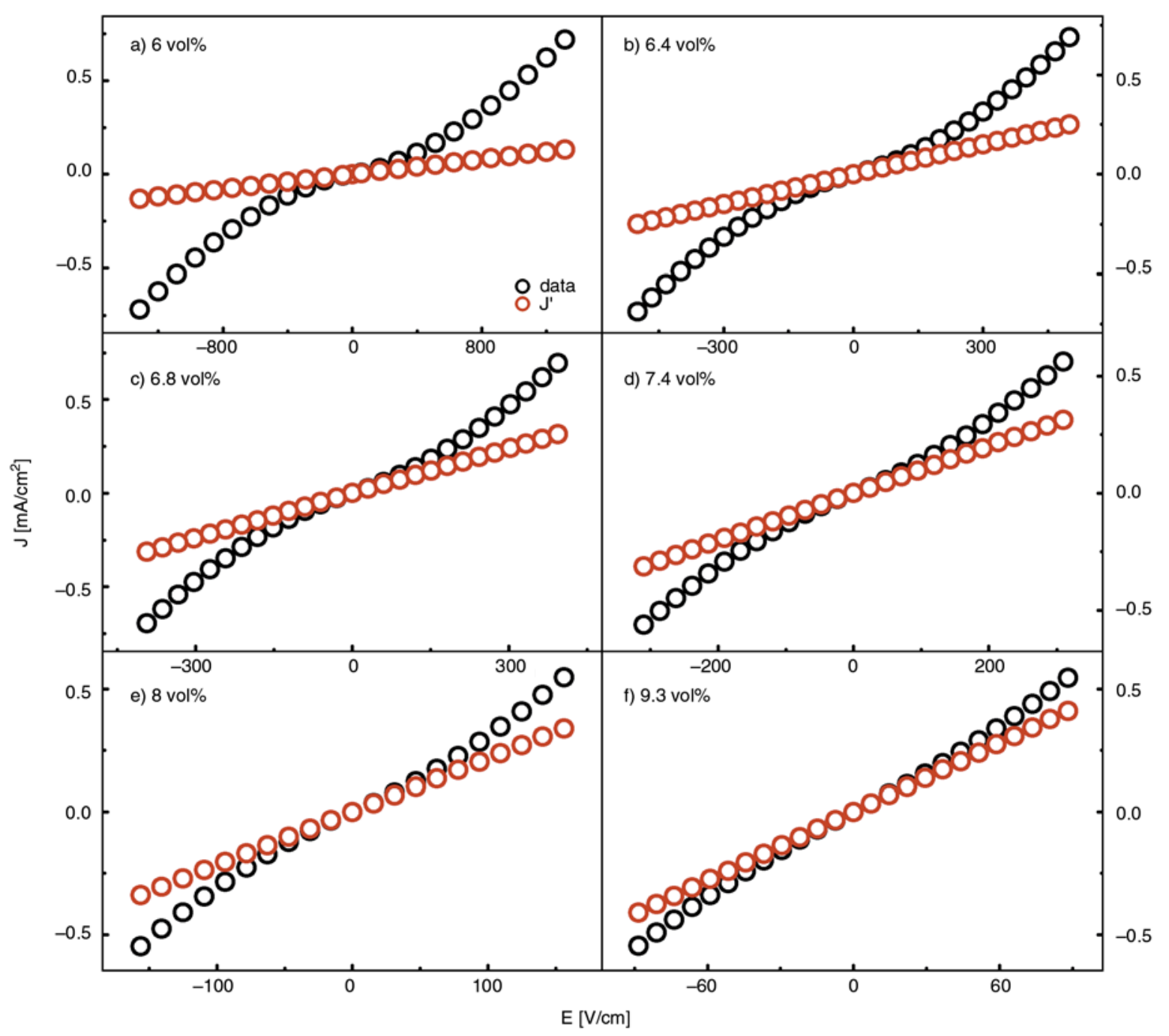

Figure 4. $J-E$ characteristics of graphite/epoxy composites with various filler contents (a-f). The black open circles are experimental data; red open circles are linear current density $J^{\prime}$ deduced from $\sigma(p) E$.

Actually, the working principle of certain electronic devices is based on this mechanism [31, 32]. Since Zener breakdown is caused by the band-to-band tunneling, thus it would not disrupt the structure of material, i.e. the $J-E$ characteristic is reversible. The current density caused by the Zener breakdown is given by Equation (2) [28]:

$$
J(E)=A E^{\mathrm{n}} \exp \left(-\frac{B}{E}\right)
$$

where $A, B$, and $n$ are constants; the value of $n$ lies usually between 1 and 3, depending on various corrections or approximations included in the approach. $\mathrm{A}$ is related to the transition frequency, i.e. the number of attempts per second made by the charge carriers to cross the barrier. $B$ is a measure of the energy barrier between the insulating matrix and the filler material. Thus the factor $\exp (-B / E)$ represents the transition probability of charge carriers between the conductive fillers and the matrix material. Although the reversible dielectric breakdown of the composites is caused by Zener tunneling as shown in Figure 4, the composite samples do not behave fully like a real Zener diode.

By separating linear $J^{\prime \prime}(\sigma(p) E)$ from the overall nonlinear current density, the remnant $J^{\prime \prime}\left(J-J^{\prime}\right)$ is obtained, as illustrated in Figure 6a. Figure 6b shows the $\ln \left(J^{\prime \prime} / E^{\mathrm{n}}\right)$ vs. $1 / E$ plot for all composite samples. The apparent linear relationship provides strong support for the occurrence of Zener breakdown. To the best of our knowledge, no other physical models can fit the experimental results well. The fitting parameters were extracted and summarized in Table 1. The normalized $J-E$ relationship is shown in Figure 6c. Similar to the case of alternating current conductivity of the conductor/insulator system, the data points for different composite samples fall into one curve. Accordingly, Zener effect 


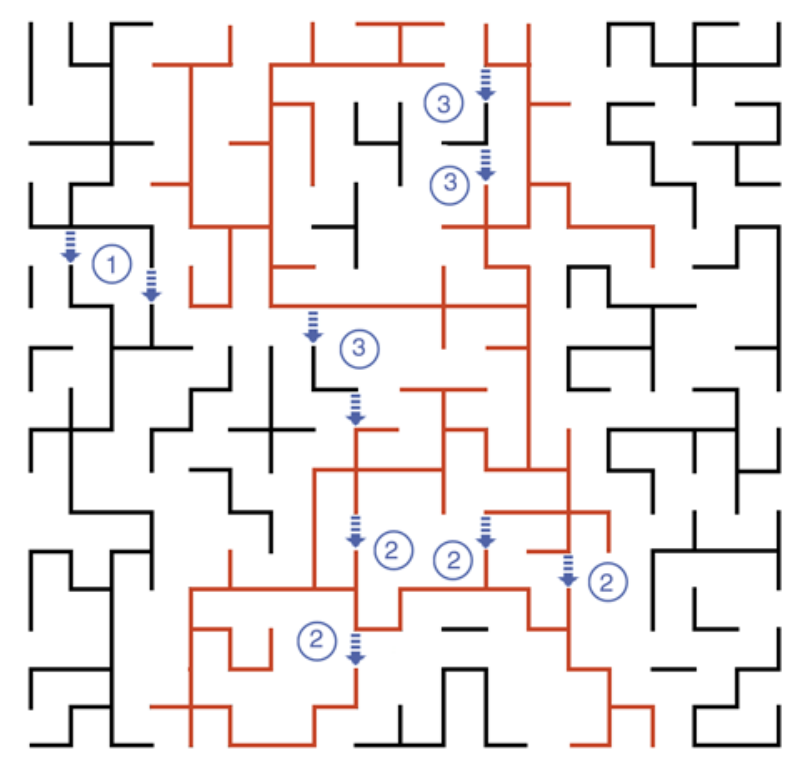

Figure 5. Two dimensional random-bond model of graphite/ epoxy system above the percolation threshold. (1), (2), (3) and (3) correspond to the occurrence of dielectric breakdown between conducting clusters, between different parts of the conducting backbone, and between the clusters and the backbone, respectively.

Table 1. Parameters characterizing Zener current for the graphite/epoxy composites

\begin{tabular}{|c|c|c|r|}
\hline $\mathbf{p}$ & $\mathbf{n}$ & $\mathbf{I n A}$ & $\mathbf{B}[\mathbf{V} / \mathbf{c m}]$ \\
\hline $6.0 \mathrm{vol} \%$ & $1.75 \pm 0.02$ & $-12.74 \pm 0.32$ & $28.69 \pm 4.21$ \\
\hline $6.4 \mathrm{vol} \%$ & $1.86 \pm 0.03$ & $-12.34 \pm 0.30$ & $17.23 \pm 3.15$ \\
\hline $6.8 \mathrm{vol} \%$ & $1.85 \pm 0.03$ & $-12.03 \pm 0.27$ & $5.52 \pm 1.32$ \\
\hline $7.4 \mathrm{vol} \%$ & $1.82 \pm 0.03$ & $-11.86 \pm 0.26$ & $4.08 \pm 1.02$ \\
\hline $8.0 \mathrm{vol} \%$ & $1.84 \pm 0.03$ & $-10.93 \pm 0.21$ & $2.64 \pm 0.34$ \\
\hline $9.3 \mathrm{vol} \%$ & $1.73 \pm 0.02$ & $-9.43 \pm 0.15$ & $8.89 \pm 1.73$ \\
\hline
\end{tabular}

reflects intrinsic properties of a certain conductor/ insulator system.

For the composites with higher graphite concentration, a larger number of charge carriers are available, causing an increase of the transition frequency of charge carriers. This is manifested by larger $A$ values. Therefore, $A$ is not only related to the filler concentration but also to the filler dispersion. In addition, the internal insulating gaps tend to become smaller with increasing graphite concentration (see Figure 1), assuming that the graphite powders are dispersed uniformly in the polymer matrix. This leads to smaller $B$ values with increasing filler content up to 8 vol\%, favoring the occurrence of Zener breakdown. However, the $B$ value rises sharply at $9.3 \mathrm{vol} \%$ graphite content. This is because the viscosity of liquid mixture during the composite processing is very large. In this case, pure stirring cannot effectively disperse graphite fillers homoge-
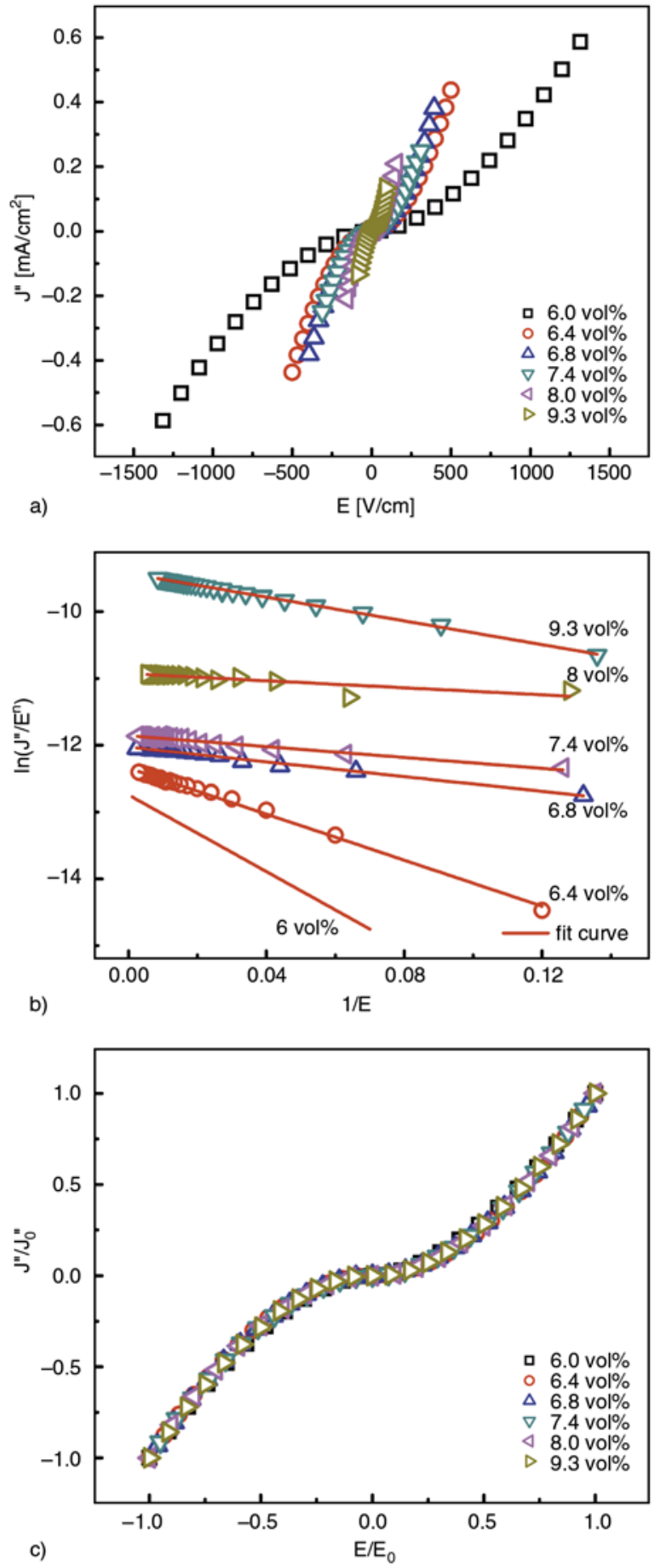

Figure 6. (a) Plot of $J^{\prime \prime}$ vs. $E$ for graphite/epoxy composite samples with various filler contents. (b) Relationship between $\ln \left(J^{\prime \prime} / E^{\mathrm{n}}\right)$ and $1 / E$, where the linear fitting results are shown in red solid lines. $\mathrm{n}$ is evaluated from the data in (a). (c) Normalized relationship of $J^{\prime \prime}$ vs. $E$. $J^{\prime \prime}{ }_{0}$ and $E_{0}$ are the maximum testing field strength and corresponding Zener current density as shown in (a).

neously in the matrix material. This results in the formation of aggregates (see Figure 1f), thereby producing higher energy barrier for the charge carriers 
to tunnel through. The aggregation also influences the static conductivity of the composites. However, the graphite/epoxy composites still exhibit percolative behavior as shown in Figure 3. Overall, Zener current tends to increase with filler content due to the presence of large amount of internal charge carriers.

As Zener breakdown relates to the band-to-band tunneling of charge carriers (from valence band to conduction band for electrons and vise versa for holes), the band gap and band tilt due to the external field are two main factors governing the generation of charge carriers, as illustrated in Figure 7. Obviously, a narrower band gap shortens the tunneling distance, facilitating interband tunneling. Similarly, a large electric field decreases the tunneling distance by tilting the band seriously. For the polymer composites, the width of the forbidden band is influenced by the nature of polymer matrix, while the internal field strength is determined by the dispersion of the conducting fillers. In order to suppress the Zener effect, the polymer matrix with a wide forbidden band is preferred (Figure 7b). Also, a poor dispersion of conductive fillers within the insulating matrix can also achieve the same results.
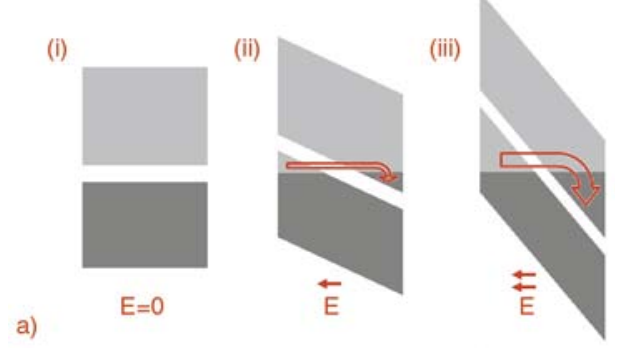

(i)

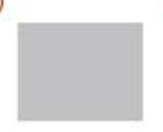

(ii)
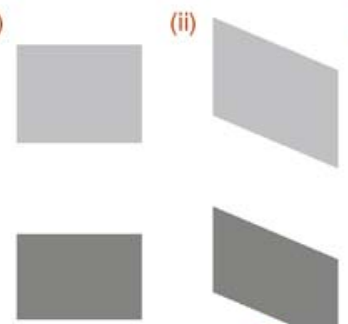

$E=0$

b)
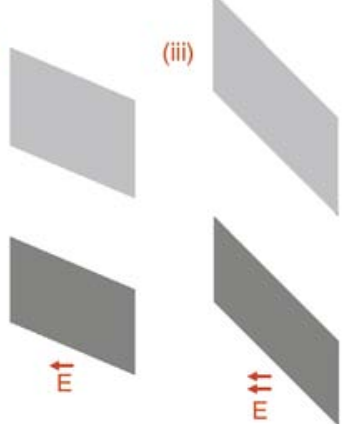

Figure 7. Transition of electrons from valence band to conduction band for insulating matrix with a (a) narrow and (b) wide forbidden band. (i), (ii), and (iii) illustrate the cases in which the insulating matrix subjected to no electric field, intermediate electric field, and large electric field, respectively.

\section{Conclusions}

In summary, nonlinear electrical transport is observed in the graphite/epoxy composites. The electrical nonlinearity is attributed to reversible dielectric breakdown inside the system. Such reversible breakdown is caused by the Zener effect, resulting from a magnified internal electric field imposed to thin insulating polymer layers. The experimental results provide strong support for the occurrence of Zener breakdown. The characteristics of Zener breakdown reveal certain important aspects relating to the electrical conduction within the composite system, such as the properties of the fillers, the dispersion of the fillers, and the nature of the polymer matrix.

\section{Acknowledgements}

This work is supported by a Strategic Grant (No. 7002772), City University of Hong Kong.

\section{References}

[1] Oh J-H., Oh K-S., Kim C-G., Hong C-S.: Design of radar absorbing structures using glass/epoxy composite containing carbon black in X-band frequency ranges. Composites Part B: Engineering, 35, 49-56 (2004). DOI: $10.1016 /$ j.compositesb.2003.08.011

[2] Arshak K., Morris D., Arshak A., Korostynska O.: Sensitivity of polyvinyl butyral/carbon-black sensors to pressure. Thin Solid Films, 516, 3298-3304 (2008). DOI: $10.1016 /$ j.tsf.2007.09.006

[3] Mrozek R. A., Cole P. J., Mondy L. A., Rao R. R., Bieg L. F., Lenhar J. L.: Highly conductive, melt processable polymer composites based on nickel and low melting eutectic metal. Polymer, 51, 2954-2958 (2010). DOI: $10.1016 /$ j.polymer.2010.04.067

[4] Psarras G. C.: Hopping conductivity in polymer matrixmetal particles composites. Composites Part A: Applied Science and Manufacturing, 37, 1545-1553 (2006). DOI: 10.1016/j.compositesa.2005.11.004

[5] Dang Z-M., Yuan J-K., Zha J-W., Zhou T., Li S-T., Hu G-H.: Fundamentals, processes and applications of high-permittivity polymer-matrix composites. Progress in Materials Science, 57, 660-723 (2012). DOI: $10.1016 /$ j.pmatsci.2011.08.001

[6] Dang Z-M., Wang L., Yin Y., Zhang Q., Lei Q-Q.: Giant dielectric permittivities in functionalized carbon-nanotube/ electroactive-polymer nanocomposites. Advanced Materials, 19, 852-857 (2007). DOI: $10.1002 /$ adma.200600703

[7] Stauffer A. D.: Introduction to percolation theory. Taylor and Francis, London (2003).

[8] Dye J. C., Schrøder T. B.: Universality of ac conduction in disordered solids. Review of Modern Physics, 72, 873-892 (2000). DOI: $10.1103 /$ RevModPhys. 72.873 
[9] Lin H., Lu W., Chen G.: Nonlinear DC conduction behavior in epoxy resin/graphite nanosheets composites. Physica B: Condensed Matter, 400, 229-236 (2007). DOI: $10.1016 /$ j.physb.2007.07.015

[10] Chen G., Weng W., Wu D., Wu C.: Nonlinear conduction in nylon-6/foliated graphite nanocomposites above the percolation threshold. Journal of Polymer Science Part B: Polymer Physics, 42, 155-167 (2004). DOI: 10.1002/polb.10682

[11] Zheng Q., Song Y., Wu G., Yi X.: Reversible nonlinear conduction behavior for high-density polyethylene/ graphite powder composites near the percolation threshold. Journal of Polymer Science Part B: Polymer Physics, 39, 2833-2842 (2001).

DOI: $10.1002 /$ polb.10042

[12] Celzard A., Furdin G., Marêché J. F., McRae E.: Nonlinear current-voltage characteristics in anisotropic epoxy resin-graphite flake composites. Journal of Materials Science, 32, 1849-1853 (1997).

DOI: 10.1023/A:1018504906935

[13] Alam M. A., Weir B. E., Silverman P. J.: A study of soft and hard breakdown - Part I: Analysis of statistical percolation conductance. IEEE Transactions on Electron Devices, 49, 232-238 (2002).

DOI: $10.1109 / 16.981212$

[14] Kim J., Grzybowski B. A.: Controlling reversible dielectric breakdown in metal/polymer nanocomposites. Advanced Materials, 24, 1850-1855 (2012). DOI: 10.1002/adma.201104334

[15] Zhou L., Lin J., Chen G.: Electrical breakdown in high-density polyethylene/graphite nanosheets conductive composites. Journal of Polymer Science Part B: Polymer Physics, 47, 576-582 (2009). DOI: $10.1002 /$ polb.21663

[16] Song Y., Shen Y., Liu H., Lin Y., Li M., Nan C-Y.: Improving the dielectric constants and breakdown strength of polymer composites: Effects of the shape of the $\mathrm{BaTiO}_{3}$ nanoinclusions, surface modification and polymer matrix. Journal of Materials Chemistry, 22, 16491-16498 (2012).

DOI: $10.1039 / C 2 J M 32579 A$

[17] Schuman T. P., Siddabattuni S., Cox O., Dogan F.: Improved dielectric breakdown strength of covalentlybonded interface polymer-particle nanocomposites. Composite Interfaces, 17, 719-731 (2010). DOI: $10.1163 / 092764410 X 495315$

[18] Sen A. K.: Nonlinear response, semi-classical percolation and breakdown in the RRTN Mode 1. in 'Lecture notes in physics: Quantum and semi-classical percolation and breakdown in disordered solids' (eds.: Chakrabarti B. K., Bardhan K. K., Sen A. K.) Springer, Heildelberg, Vol 762, 1-62 (2009).

[19] Gupta A. K., Sen A. K.: Nonlinear dc response in composites: A percolative study. Physical Review B, 57, 3375-3388 (1998).

DOI: 10.1103/PhysRevB.57.3375
[20] He L. X., Tjong S-C.: Universality of Zener tunneling in carbon/polymer composites. Synthetic Metals, 161, 2647-2650 (2012).

DOI: 10.1016/j.synthmet.2011.09.037

[21] Song Y. S., Youn J. R.: Influence of dispersion states of carbon nanotubes on physical properties of epoxy nanocomposites. Carbon, 43, 1378-1385 (2005).

DOI: $10.1016 /$ j.carbon.2005.01.007

[22] Balberg I.: A comprehensive picture of the electrical phenomena in carbon black-polymer composites. Carbon, 40, 139-143 (2002).

DOI: 10.1016/S0008-6223(01)00164-6

[23] Ezquerra T. A., Kulescza M., Cruz C. S., Baltá-Calleja F. J.: Charge transport in polyethylene-graphite composite materials. Advanced Materials, 2, 597-600 (1990). DOI: 10.1002/adma.19900021209

[24] Mamunya Y. P., Muzychenko Y. V., Pissis P., Lebedev E. V., Shut M. I.: Percolation phenomena in polymers containing dispersed iron. Polymer Engineering and Science, 42, 90-100 (2002).

DOI: $10.1002 /$ pen. 10930

[25] Logakis E., Pandis Ch., Peoglos V., Pissis P., Pionteck J., Pötschke P., Mičušík M., Omastová M.: Electrical/ dielectric properties and conduction mechanism in melt processed polyamide/multi-walled carbon nanotubes composites. Polymer, 50, 5103-5111 (2009)

DOI: $10.1016 /$ j.polymer.2009.08.038

[26] Gefen Y., Shih W-H., Laibowitz R. B., Viggiano J. M.: Nonlinear behavior near the percolation metal-insulator transition. Physical Review Letters, 57, 3097-3100 (1986).

DOI: 10.1103/PhysRevLett.57.3097

[27] Sheng P., Sichel E. K., Gittleman J. I.: Fluctuationinduced tunneling conduction in carbon-polyvinylchloride composites. Physical Review Letters, 40, 11971200 (1978).

DOI: $10.1103 /$ PhysRevLett.40.1197

[28] Zener C.: A theory of the electrical breakdown of solid dielectrics. Proceedings of Royal Society A, 145, 523539 (1934).

DOI: $10.1098 /$ rspa.1934.0116

[29] Chynoweth A. G.: Progress in semiconductors. John Wiley and Sons, New York (1960).

[30] McAfee K. B., Ryder E. J., Shockley W., Sparks M.: Observations of Zener current in germanium p-n junctions. Physical Review, 83, 650-651 (1951).

DOI: 10.1103/PhysRev.83.650

[31] Kleemann H., Gutierrez R., Lindner F., Avdoshenko S., Manrique P. D., Lüssem B., Cuniberti G., Leo K.: Organic Zener diodes: Tunneling across the gap in organic semiconductor materials. Nano Letters, 10, 4929-4934 (2010).

DOI: $10.1021 / \mathrm{nl} 102916 \mathrm{n}$

[32] Reddick W. M., Amaratunga G. A. J.: Silicon surface tunnel transistor. Applied Physics Letters, 67, 494-496 (1995). DOI: $10.1063 / 1.114547$ 